\section{Atenção pré-natal na cidade de Pelotas, Rio Grande do Sul, Brasil}

\section{Prenatal care in the city of Pelotas, in the State of Rio Grande do Sul, Brazil}

Isabel Cristina Rosa Barros Rasia 1

Elaine Albernaz 2

1,2 Programa de Pós-Graduação em Saúde e Comportamento. Universidade Católica de Pelotas. Rua Felix da Cunha, 412. Pelotas, RS, Brasil. CEP: 96.020-220. E-mail: irasia@ig.com.br

\section{Abstract}

Objectives: to identify key features of prenatal care in the city of Pelotas, in the State of Rio Grande do Sul, Brazil.

Methods: a cross-sectional study was conducted with a cohort of 2741 mothers, interviewed in the city's clinics, between September 2002 and May 2003. The mothers included answered a standard questionnaire which provided data regarding demographic and socioeconomic factors as well as aspects relating to prenatal care and the clinics attended. The outcome was considered to be either proper or improper prenatal care, the criterion being the number of medical consultations, with six or more appointments constituting adequate prenatal care.

Results: it was observed that $77 \%$ of the expectant mothers carried out six or more prenatal consultations, but there were shortcomings in terms of the success and management of prenatal care, such as treatment of gynecological problems and education. The risk of not having proper prenatal care was higher among black or mixed-race expectant mothers $(R O=1.7)$, with fewer years of schooling $(R O=3.3)$ and lower income $(R O=3.0)$. This was also the case with expectant mothers without partners $(R O=2.0)$, and smokers $(R O=1.5)$.

Conclusions: the results are consistent with those found in the literature, which depict an inversion of the needed pattern of care. Patients with the worse economic conditions and the least schooling, run a higher risk of receiving inadequate prenatal care and of being subject to the consequences of this.

Key words Prenatal care, Quality of health care

\section{Resumo}

Objetivos: identificar as características da assistência pré-natal na da cidade de Pelotas, Rio Grande do Sul, Brasil.

Métodos: estudo transversal aninhado a uma coorte de 2741 mães, entrevistadas nas maternidades da cidade, no periodo de setembro de 2002 a maio de 2003. As pacientes incluidas responderam a um questionário padronizado que obteve dados referentes a fatores demográficos e socioeconômicos e características do pré-natal. O desfecho analisado foi pré-natal adequado ou inadequado conforme o número de consultas, sendo definido como adequado, a realização de seis ou mais consultas.

Resultados: observou-se que $77 \%$ das gestantes fizeram seis ou mais consultas de pré-natal, porém, existem falhas no cumprimento e no manejo das normas de assistência pré-natal, como tratamento de problemas ginecológicos e orientações educativas. $O$ risco de não realizar um pré-natal adequado foi maior para as gestantes de cor negra ou parda $(R O=1,7)$, com menor escolaridade $(R O=3,3) e$ menor renda $(R O=3,0)$; assim como para as gestantes sem companheiro $(R O=2,0)$ e fumantes $(R O=1,5)$.

Conclusões: os resultados condizem com os achados na literatura, na qual existe uma inversão de cuidados. As pacientes com piores condições econômicas e pouca escolaridade apresentam um maior risco para a realização de um pré-natal inadequado e suas conseqüencias.

Palavras-chave Cuidado pré-natal, Qualidade da assistência à saúde 


\section{Introdução}

Os serviços de atendimento pré-natal foram iniciados no Brasil nos anos 20-30, no pós-guerra, quando se estabeleceram como serviço indispensável para o acompanhamento da gestação. ${ }^{1}$

O controle pré-natal, segundo recomendações de organismos oficiais de saúde, deve ter início precoce, ter cobertura universal, ser realizado de forma periódica, estar integrado às demais ações preventivas e curativas, necessitando ser efetuado um número mínimo de seis consultas. ${ }^{1}$ Além disso, precisa incluir a realização de procedimentos clínicos indispensáveis para o acompanhamento da gravidez, como exames laboratoriais, vacinas e atividades educativas. 2,3

Gestantes que freqüentam serviços de atenção pré-natal apresentam menos doenças e seus filhos têm um melhor crescimento intra-uterino, menor mortalidade perinatal e infantil. O número de consultas realizadas durante o pré-natal também está diretamente relacionado a melhores indicadores de saúde materno-infantil.1,2

Os resultados de um estudo de coorte, realizado na cidade de Pelotas, Rio Grande do Sul, em 1993, mostraram que, apesar da alta cobertura pré-natal no município, existem desigualdades nos cuidados oferecidos às gestantes. As de maior risco gestacional apresentaram maior proporção de pré-natal classificado como inadequado ou intermediário (25\%), quando comparadas com as de menor risco (10\%). Aquelas sem nenhum atendimento antes do parto eram mais pobres, adolescentes ou com idade acima de 40 anos e seus bebês tiveram uma incidência de baixo peso ao nascer 2,5 vezes maior do que as do grupo de mães com cinco ou mais consultas realizadas. 4

Os procedimentos menos referidos pelas gestantes como realizados na consulta pré-natal, na coorte de Pelotas em 1993, foram exame ginecológico e orientação para amamentar, sendo avaliação da data da última menstruação, verificação do peso, medição da altura uterina e aferição da pressão arterial os mais executados. ${ }^{4-7}$ Existem ainda muitas questões na assistência pré-natal sem respostas completas quanto à freqüência, à especificidade das intervenções envolvidas e a seu conteúdo, na tentativa de definição de padrões.

Geralmente, as investigações da atenção ao prénatal têm como objetivo subsidiar ações de planejamento, monitorar a tendência na utilização de cuidados e entender a sua relação com os resultados obtidos. Poucos estudos exploram a qualidade do cuidado, que pode ser expressa pela execução adequada de intervenções que exigem serem comparadas com um padrão, muitas vezes não definido por consenso, dificultando a operacionalização das investigações e a comparação dos achados. 5

Barros et al.,8 ao compararem as coortes de nascimentos de 1982, 1993 e 2004, observaram que a prevalência de nascimentos prematuros aumentou de $6,3 \%$ em 1982 para $16,2 \%$ em 2004. O estudo também mostrou progressos nas características maternas, como peso e altura da mãe, idade, escolaridade e renda, nas coortes seguintes. Contudo, o coeficiente de mortalidade infantil aumentou, reforçando a hipótese de estarem deficientes a assistência pré-natal recebida durante a gestação e o parto. Tal estudo sugere a investigação da qualidade do cuidado oferecido às gestantes durante o prénatal, a fim de identificar os problemas.

O presente trabalho busca identificar as características da assistência pré-natal em Pelotas, avaliando número de consultas durante a gestação, realização de exames de rotina, procedimentos e orientações mínimas indispensáveis durante a gravidez.

\section{Métodos}

Esta pesquisa faz parte de um estudo longitudinal que acompanhou os recém-nascidos da zona urbana e rural na cidade de Pelotas, Rio Grande do Sul, Brasil, nascidos entre setembro de 2002 e maio de 2003. O estudo teve um componente perinatal (visita hospitalar) e outro de acompanhamento dos bebês com um, três e seis meses, a fim de avaliar aspectos relacionados à alimentação infantil e ao comportamento feminino no puerpério.

Para esse estudo, foram utilizadas informações do componente perinatal, durante a visita hospitalar, constituindo-se um delineamento transversal aninhado a um estudo de coorte, o qual teve como objetivo avaliar as características da atenção pré-natal das parturientes da amostra.

Foram entrevistadas as mães com parto ocorrido nas maternidades da cidade ou nelas atendidas depois do parto, no período de 16 de setembro de 2002 a 31 de maio de 2003. Foram considerados, como critérios de exclusão, mães soropositivas para HIV, recém-nascidos com malformações congênitas graves que impossibilitassem a amamentação, ou com domicílio fora do município de Pelotas. A amostra baseou-se na estimativa da ocorrência de 400 partos por mês, sendo a amostra final esperada de 3400 partos. 
O tamanho da amostra do estudo de coorte de Pelotas baseou-se em um intervalo de confiança de $95 \%$ e um poder estatístico de $90 \%$, com exposições variando entre 15 e $80 \%$, estimando um risco relativo (RR) de 2,0. Foram acrescentados $30 \%$ para controle de fatores de confusão em potencial e mais $10 \%$ para possíveis perdas.

Participaram do estudo todas as crianças nascidas vivas nos hospitais ou em domicílios, referenciadas para algum dos hospitais e cujas mães residissem na cidade de Pelotas. As mães incluídas responderam a um questionário padronizado, com questões fechadas e abertas, previamente testado, com informações referentes a fatores demográficos e socioeconômicos, características do pré-natal, características da mãe, do bebê e da maternidade.

O trabalho de campo foi realizado por uma equipe de 15 entrevistadores, estudantes de cursos da área da saúde, previamente treinados, sem conhecimento dos objetivos do estudo, e por uma supervisora.

As entrevistas foram feitas respeitando um período mínimo de quatro horas após o parto. As perdas que porventura ocorressem, eram anotadas e buscadas no domicílio da paciente. A supervisora foi responsável pela revisão dos questionários, codificados pelos próprios entrevistadores, e pelo cálculo da idade gestacional, data da última menstruação (DUM) e ultra-sonografia, assim como pela codificação das perguntas abertas. $\mathrm{O}$ estudo piloto foi realizado na maternidade do Hospital Universitário São Francisco de Paula (HUSFP).

A base de dados utilizada para a digitação dos dados foi o programa Epi-Info 6.0, com dupla digitação dos questionários, para comparação e detecção de possíveis erros. Quanto à análise dos dados, o programa empregado foi o SPSS for Windows. Essa análise incluiu o cálculo da freqüência das variáveis e análise bivariada entre os fatores de exposição e o desfecho, entre os fatores de exposição e outras variáveis e entre o desfecho e outras variáveis.

$\mathrm{Na}$ análise multivariada, conforme modelo hierárquico, foram mantidas, em cada nível, as variáveis com nível de significância de $p \leq 0,20$, a fim de avaliar o efeito de possíveis fatores de confusão. Foram definidas como associações estatisticamente significativas aquelas cujo valor de $p$ foi $<0,05$. O modelo hierárquico considerou a relação entre as possíveis exposições e entre estas com o desfecho. No primeiro nível do modelo foram incluídos os fatores demográficos (idade e cor) e os fatores socioeconômicos, como renda familiar (em salários mínimos), escolaridade da gestante e do companheiro (em anos completos). No segundo nível encontram-se as características maternas, como trabalho externo, problemas ginecológicos, fumo (definido como fumar pelo menos um cigarro por dia, independente do trimestre).

O desfecho foi definido como pré-natal adequado conforme o número de consultas pré-natais. Utilizou-se a normatização do Ministério da Saúde, 1 que define como pré-natal adequado a realização de seis ou mais consultas nesse período.

O controle de qualidade do estudo ocorreu por meio dos seguintes mecanismos: questionários padronizados, estudo piloto, manual de instruções (com orientações para entender, formular e codificar cada questão isoladamente), treinamento dos entrevistadores, revisão dos questionários e aplicação de um questionário sintetizado a uma amostra aleatória de $10 \%$ de todas as mães, visando avaliar a qualidade e a veracidade dos dados coletados. Igualmente todas as mães incluídas no estudo, após terem sido devidamente esclarecidas sobre a pesquisa, assinaram termo de consentimento.

O projeto de pesquisa foi aprovado pelas comissões de Pesquisa e Ética em Saúde da Santa Casa de Misericórdia de Pelotas e da Fundação de Apoio Universitário (FAU). A pesquisa também foi aprovada pelo Comitê de Ética em Pesquisa da Universidade Federal do Rio Grande do Sul (UFRGS) e Comissão Científica da Universidade Católica de Pelotas (UCPEL).

\section{Resultados}

Em Pelotas, de setembro de 2002 a maio de 2003, ocorreram 3449 nascimentos. Destes, 2799 (81\%) das mães residiam na cidade, e 29 nascimentos ocorreram em domicílios ou outro local, mas fizeram parte do estudo pois foram referenciadas para um dos hospitais da cidade para atendimento.

A pesquisa teve $2,1 \%$ de perdas e/ou recusas, sendo a amostra final estudada de 2741 mulheres.

Cerca de $32 \%$ da amostra possuía trabalho remunerado e $83 \%$ vivia com companheiro. A Tabela 1 mostra a distribuição da amostra conforme as características demográficas, socioeconômicas, maternas e do núcleo familiar. A maioria das mães eram jovens, de cor branca, com renda familiar de até três salários mínimos; $43 \%$ das gestantes estudaram entre cinco e oito anos. Quanto ao número de filhos, observou-se que $42 \%$ eram primíparas, $77 \%$ fizeram seis ou mais consultas de pré-natal e $24 \%$ haviam fumado durante a gravidez.

A Tabela 2 mostra as solicitações de exames e os procedimentos realizados durante a consulta de pré- 
Tabela 1

Distribuição da amostra conforme características demográficas, socioeconômicas e reprodutivas. Pelotas, Rio Grande do Sul, 2003.

\begin{tabular}{|c|c|c|}
\hline Características maternas $(\mathrm{N}=\mathbf{2 7 4 1})$ & $\mathbf{n}$ & $\%$ \\
\hline \multicolumn{3}{|l|}{ Idade (em anos completos) } \\
\hline$<20$ & 558 & 20,4 \\
\hline $20-34$ & 1815 & 66,2 \\
\hline$\geq 35$ & 368 & 13,4 \\
\hline \multicolumn{3}{|l|}{ Cor } \\
\hline Branca & 2040 & 74,4 \\
\hline Não-branca & 701 & 25,6 \\
\hline \multicolumn{3}{|l|}{ Renda familiar (em salários mínimos) } \\
\hline Até 1 & 606 & 22,1 \\
\hline $1,1-3$ & 1293 & 47,2 \\
\hline $3,1-6$ & 532 & 19,4 \\
\hline$>6$ & 310 & 11,3 \\
\hline \multicolumn{3}{|c|}{ Escolaridade da gestante (em anos completos) } \\
\hline $0-4$ & 532 & 19,4 \\
\hline $5-8$ & 1183 & 43,2 \\
\hline 9 ou mais & 1026 & 37,4 \\
\hline \multicolumn{3}{|c|}{ Escolaridade do companheiro (em anos completos) } \\
\hline $0-4$ & 468 & 17,1 \\
\hline $5-8$ & 1390 & 50,7 \\
\hline 9 ou mais & 883 & 32,2 \\
\hline \multicolumn{3}{|l|}{ Paridade } \\
\hline 1 & 1170 & 41,7 \\
\hline $2-4$ & 1356 & 50,2 \\
\hline$\geq 5$ & 215 & 8,1 \\
\hline \multicolumn{3}{|l|}{ Fumo } \\
\hline Sim & 643 & 23,5 \\
\hline Não & 2098 & 76,5 \\
\hline \multicolumn{3}{|l|}{ Número de consultas de pré-natal } \\
\hline Nenhuma & 60 & 2,2 \\
\hline $1-5$ & 567 & 20,7 \\
\hline$\geq 6$ & 2114 & 77,1 \\
\hline
\end{tabular}

natal. Procedimentos como solicitações de ultrasonografia, medida da pressão arterial e ausculta do bebê foram efetuados em quase todas as gestantes. Cerca de $86 \%$ das pacientes fizeram imunização antitetânica e $78 \%$ tomou vitaminas (ferro). Além disso, exames de sangue, de urina, aferição do peso da gestante foram realizados em quase toda a amostra.

A altura da gestante foi medida em $65 \%$ da amostra e a altura uterina em $96 \%$; metade das pacientes referiu presença de corrimento, embora apenas $33 \%$ tenham sido examinadas por esse motivo. Exame das mamas foi realizado em somente $53 \%$ das gestantes, pré-câncer em $31 \%$. Teste de tolerância à glicose foi efetuado em $38 \%$ das gestantes, 29\% das gestantes tomaram antibióticos e um terço das mães tomou outros remédios durante a gestação.

Orientações sobre amamentação e parto foram repassadas apenas a $48 \%$ e $61 \%$ das gestantes respectivamente. Registros das consultas na carteira da gestante foram realizados em $88 \%$ da amostra.

A Tabela 3 compara os fatores associados ao número de consultas no pré-natal. Observa-se que as gestantes adolescentes, de cor negra ou parda, com menor renda e escolaridade apresentaram um risco maior de realizarem um pré-natal inadequado. Para as variáveis renda e escolaridade, evidenciou-se um efeito dose-resposta.

As gestantes sem companheiro, fumantes e sem trabalho remunerado, tiveram um risco cerca de duas vezes maior de não realizarem um pré-natal 
Tabela 2

Prevalência de solicitação de exames e procedimentos realizados na consulta pré-natal. Pelotas, Rio Grande do Sul, 2003.

\begin{tabular}{|c|c|c|}
\hline Variáveis ( $\mathrm{N}=2741)$ & $\mathbf{n}$ & $\%$ \\
\hline \multicolumn{3}{|l|}{ Exame de urina } \\
\hline Sim & 2694 & 98,3 \\
\hline Não & 47 & 1,7 \\
\hline \multicolumn{3}{|l|}{ Ultrassonografia } \\
\hline Sim & 2648 & 96,6 \\
\hline Não & 93 & 3,4 \\
\hline \multicolumn{3}{|l|}{ Vacina antitetânica } \\
\hline Sim & 2355 & 85,9 \\
\hline Não & 386 & 14,1 \\
\hline \multicolumn{3}{|l|}{ Exame das mamas } \\
\hline Sim & 1441 & 52,6 \\
\hline Não & 1300 & 47,4 \\
\hline \multicolumn{3}{|l|}{ Toque vaginal } \\
\hline $\operatorname{Sim}$ & 653 & 23,8 \\
\hline Às vezes & 1559 & 56,9 \\
\hline Nunca & 529 & 19,3 \\
\hline \multicolumn{3}{|l|}{ Pré-câncer } \\
\hline Sim & 864 & 31,5 \\
\hline Não & 1877 & 68,5 \\
\hline \multicolumn{3}{|l|}{ Altura uterina } \\
\hline Sim & 2639 & 96,4 \\
\hline Às vezes & 78 & 2,8 \\
\hline Nunca & 24 & 0,8 \\
\hline \multicolumn{3}{|l|}{ Pressão arterial } \\
\hline Sim & 2713 & 99,0 \\
\hline Às vezes & 27 & 1,0 \\
\hline Nunca & 1 & 0,0 \\
\hline \multicolumn{3}{|c|}{ Orientação médica sobre o trabalho de parto } \\
\hline Sim & 1669 & 60,9 \\
\hline Não & 1072 & 39,1 \\
\hline \multicolumn{3}{|c|}{ Registro da consulta na carteira da gestante } \\
\hline Sim & 2411 & 88,0 \\
\hline Não & 330 & 12,0 \\
\hline \multicolumn{3}{|c|}{ Prescrição de ferro durante o pré-natal } \\
\hline Sim & 2132 & 77,8 \\
\hline Não & 609 & 22,2 \\
\hline \multicolumn{3}{|c|}{ Ausculta de batimentos cárdio-fetias nas consultas } \\
\hline Sim & 2681 & 97,8 \\
\hline Às vezes & 55 & 2,0 \\
\hline Nunca & 5 & 0,2 \\
\hline \multicolumn{3}{|c|}{ Corrimento durante a gestação } \\
\hline Sim & 1369 & 49,9 \\
\hline Não & 1372 & 50,1 \\
\hline \multicolumn{3}{|c|}{ Realização de exame pelo corrimento } \\
\hline Sim & 914 & 33,3 \\
\hline Não & 1827 & 66,7 \\
\hline \multicolumn{3}{|c|}{ Orientações sobre amamentação } \\
\hline Sim & 1324 & 48,3 \\
\hline Não & 1417 & 51,7 \\
\hline \multicolumn{3}{|c|}{ Teste de tolerância à glicose } \\
\hline Sim & 1048 & 38,2 \\
\hline Não & 1693 & 61,8 \\
\hline
\end{tabular}


Tabela 3

Fatores associados ao número de consultas pré-natal. Pelotas, Rio Grande Sul, 2003.

\begin{tabular}{|c|c|c|c|c|c|c|}
\hline \multirow[t]{3}{*}{ Variável ( $\mathrm{N}=2114)$} & \multicolumn{4}{|c|}{ Pré-natal* } & \multirow[t]{3}{*}{$\operatorname{RR}(\operatorname{IC} 95 \%) * *$} & \multirow[t]{3}{*}{ Valor $p$} \\
\hline & \multicolumn{2}{|c|}{ Inadequado } & \multicolumn{2}{|c|}{ Adequado } & & \\
\hline & $\mathrm{n}$ & $\%$ & $\mathrm{n}$ & $\%$ & & \\
\hline \multicolumn{7}{|c|}{ Idade (em anos completos) } \\
\hline$<20$ & 167 & 26,63 & 391 & 18,50 & $1,38(1,09-1,74)$ & $<0,001$ \\
\hline $20-34$ & 380 & 60,61 & 1435 & 67,88 & $0,96(0,78-1,19)$ & \\
\hline$\geq 35$ & 80 & 12,76 & 288 & 13,62 & 1,00 & \\
\hline \multicolumn{7}{|l|}{ Cor } \\
\hline Branca & 381 & 60,77 & 1659 & 78,48 & 1,00 & $<0,001$ \\
\hline Não-branca & 246 & 39,23 & 455 & 21,52 & $1,88(1,64-2,15)$ & \\
\hline \multicolumn{7}{|c|}{ Renda familiar (em salários mínimos) } \\
\hline $1,1-3$ & 291 & 46,41 & 1002 & 47,40 & $3,17(2,09-4,80)$ & \\
\hline $3,1-6$ & 71 & 11,32 & 461 & 21,81 & $1,88(1,19-2,97)$ & \\
\hline$>6$ & 22 & 3,51 & 288 & 13,62 & 1,00 & \\
\hline \multicolumn{7}{|c|}{ Escolaridade da gestante (em anos) } \\
\hline $0-4$ & 209 & 33,33 & 323 & 15,28 & $4,43(3,54-5,53)$ & $<0,001$ \\
\hline $5-8$ & 327 & 52,15 & 856 & 40,49 & $3,12(2,51-3,87)$ & \\
\hline 9 ou mais & 91 & 14,52 & 935 & 44,23 & 1,00 & \\
\hline \multicolumn{7}{|c|}{ Escolaridade do companheiro (em anos) } \\
\hline $0-4$ & 171 & 27,27 & 297 & 14,05 & $3,58(2,85-4,51)$ & $<0,001$ \\
\hline $5-8$ & 366 & 58,38 & 1024 & 48,44 & $2,58(2,08-3,20)$ & \\
\hline 9 ou mais & 90 & 14,35 & 793 & 37,51 & 1,00 & \\
\hline \multicolumn{7}{|l|}{ Trabalho externo } \\
\hline $\operatorname{sim}$ & 144 & 22,97 & 743 & 35,15 & 1,00 & $<0,001$ \\
\hline 1 & 164 & 26,16 & 979 & 46,31 & 1,00 & $<0,001$ \\
\hline $2-4$ & 350 & 55,82 & 1025 & 48,49 & $1,77(1,50-2,10)$ & \\
\hline$\geq 5$ & 113 & 18,02 & 110 & 5,20 & $3,53(2,91-4,28)$ & \\
\hline \multicolumn{7}{|l|}{ Companheiro } \\
\hline $\operatorname{sim}$ & 456 & 72,73 & 1824 & 86,28 & 1,00 & $<0,001$ \\
\hline Não & 171 & 27,27 & 290 & 13,72 & $1,85(1,61-2,14)$ & \\
\hline \multicolumn{7}{|l|}{ Fumo } \\
\hline $\operatorname{Sim}$ & 241 & 38,44 & 402 & 19,02 & $2,04(1,78-2,33)$ & $<0,001$ \\
\hline Não & 386 & 61,56 & 1712 & 80,98 & 1,00 & \\
\hline
\end{tabular}

* Pré-natal adequado: $\geq 6$ consultas; **RR (IC95\%)=risco relativo e intervalo de confiança de $95 \%$, para a realização de menos de seis consultas.

adequado. Para as grandes multíparas, o risco foi três vezes maior.

A análise multivariada (Tabela 4) mostra que, após serem ajustadas para outras variáveis do primeiro nível, apenas a variável trabalho externo não se manteve associado ao desfecho $(p=0,37)$. Ao serem ajustadas para as variáveis do mesmo nível e do anterior, observou-se que as demais variáveis tiveram sua medida de risco diminuída, mas mantiveram-se associadas ao desfecho, demonstrando que parte de seu poder explicativo era devido a fatores de confusão.

A paridade mostrou-se fortemente associada com o desfecho, indicando que para a grande multípara, aumentaram as chances de realização de um prénatal inadequado em cerca de cinco vezes. 
Análise multivariada dos fatores associados a pré-natal inadequado. Pelotas, Rio Grande do Sul, 2003.

\begin{tabular}{|c|c|c|c|}
\hline Variável & Rob (IC95\%)* & Roa $($ IC95\%)** & valor $p$ \\
\hline \multicolumn{4}{|c|}{ Idade (em anos completos) ${ }^{* * *}$} \\
\hline$<20$ & $1,54(1,13-2,09)$ & $1,38(0,99-1,91)$ & \multirow[t]{3}{*}{$<0,03$} \\
\hline $20-34$ & $0,95(0,73-1,25)$ & $1,02(0,76-1,37)$ & \\
\hline$\geq 35$ & 1,00 & 1,00 & \\
\hline \multicolumn{4}{|l|}{ Cor*** } \\
\hline Branca & 1,00 & 1,00 & \multirow[t]{2}{*}{$<0,001$} \\
\hline Não branca & $2,35(1,94-2,85)$ & $1,69(1,38-2,07)$ & \\
\hline \multicolumn{4}{|c|}{ Renda familiar (em salários mínimos)*** } \\
\hline Até 1 & $8,76(5,51-13,92)$ & $2,95(1,79-4,88)$ & \multirow[t]{4}{*}{$<0,001$} \\
\hline $1,1-3$ & $3,80(2,42-5,98)$ & $1,33(1,07-2,79)$ & \\
\hline $3,1-6$ & $2,01(1,22-3,32)$ & $1,35(0,80-2,27)$ & \\
\hline$>6$ & 1,00 & 1,00 & \\
\hline \multicolumn{4}{|c|}{ Escolaridade da gestante (em anos)*** } \\
\hline $0-4$ & $6,65(5,04-8,77)$ & $3,28(2,39-4,49)$ & \multirow[t]{3}{*}{$<0,001$} \\
\hline $5-8$ & $3,92(3,05-5,04)$ & $2,38(1,81-3,12)$ & \\
\hline 9 ou mais & 1,00 & 1,00 & \\
\hline \multicolumn{4}{|c|}{ Escolaridade do companheiro (em anos)*** } \\
\hline $0-4$ & $5,07(3,80-6,77)$ & $2,07(1,49-2,87)$ & \multirow[t]{2}{*}{$<0,001$} \\
\hline $5-8$ & $3,15(2,46-4,04)$ & $1,65(1,26-2,17)$ & \\
\hline 9 ou mais & 1,00 & 1,00 & \\
\hline \multicolumn{4}{|c|}{ Trabalho externo**** } \\
\hline Sim & 1,00 & 1,00 & \multirow[t]{2}{*}{0,37} \\
\hline Não & $1,82(1,48-2,23)$ & $1,11(0,88-1,42)$ & \\
\hline \multicolumn{4}{|l|}{ Paridade $* * * *$} \\
\hline 1 & 1,00 & 1,00 & \multirow[t]{3}{*}{$<0,001$} \\
\hline $2-4$ & $2,04(1,66-2,50)$ & $2,10(1,62-2,73)$ & \\
\hline$\geq 5$ & $6,13(4,49-8,36)$ & $4,80(3,23-7,11)$ & \\
\hline \multicolumn{4}{|c|}{ Companheiro**** } \\
\hline Sim & 1,00 & 1,00 & \multirow[t]{2}{*}{$<0,001$} \\
\hline Não & $2,36(1,90-2,92)$ & $2,14(1,67-2,73)$ & \\
\hline \multicolumn{4}{|l|}{ Fumo**** } \\
\hline Sim & $2,66(2,19-3,23)$ & $1,52(1,23-1,89)$ & \multirow[t]{2}{*}{$<0,001$} \\
\hline Não & 1,00 & 1,00 & \\
\hline
\end{tabular}

* Rob=razão de odds bruta e intervalo de confiança de $95 \%$; ** Roa=razão de odds ajustada e intervalo de confiança de $95 \%$; *** Modelo I-cor, idade, renda familiar, escolaridade da gestante e do companheiro; *** Modelo II-cor, idade, renda familiar, escolaridade da gestante e do companheiro, trabalho externo, paridade, presença de companheiro e fumo.

\section{Discussão}

Segundo recomendações do Ministério da Saúde, ${ }^{1}$ toda gestante tem direito a atendimento digno e de qualidade no decorrer da gestação, parto e puerpério. Informações epidemiológicas com a finalidade de avaliar a efetividade de ações e serviços assistenciais são essenciais para a racionalidade e a qualidade dos sistemas de saúde. 7

Entre as limitações deste estudo, destaca-se o fato da aplicação de um instrumento não específico para o pré-natal, pois o estudo está aninhado a uma coorte, não contemplando a idade gestacional inicial no questionário aplicado às mães. Embora, muitas pesquisas que avaliam a assistência pré-natal também não forneçam informações sobre o conteúdo, continuidade e qualidade da assistência prestada; apesar de as informações sobre a extensão do cuidado serem importantes e evidenciarem os problemas enfrentados e as deficiências do sistema de saúde. 5-7

O presente estudo mostra as características da 
assistência pré-natal de Pelotas, e revela que mais de $77 \%$ das gestantes fizeram seis ou mais consultas de pré-natal. Várias características maternas avaliadas foram semelhantes aos achados de outros estudos realizados na cidade, principalmente estudos de coortes que acompanharam mães e bebês em 1982 e $1993.5,6,9$

Apesar da alta cobertura pré-natal, confirmaramse os achados anteriores, na revisão da literatura, descrevendo a desigualdade dos cuidados oferecidos à gestante. As mães com melhores condições socioeconômicas e maior escolaridade continuam sendo as que mais consultam, evidenciando a desigualdade social, em que os grupos sociais mais vulneráveis recebem atenção pré-natal deficiente, obedecendo à lei dos cuidados inversos. 8,10

Foi observada uma alta incidência de mães fumantes, na razão de uma para cada quatro. Outras pesquisas verificaram que o tabagismo materno aumenta o risco de crianças pequenas para idade gestacional (PIG), afetando o crescimento intrauterino.11,12 O Centro Brasileiro de Informações Sobre Drogas Psicotrópicas (CEBRID) 13 alerta que, na gravidez, o feto passa a receber as substâncias tóxicas através da placenta. A nicotina provoca aumento de batimentos cardíacos no feto, redução de peso ao nascer, menor estatura, além de alterações neurológicas importantes. O risco de abortamento espontâneo é maior em gestantes que fumam. Orientações contra o fumo na gravidez devem ser intensificadas nas consultas de pré-natal.

Apesar de o número de consultas pré-natais estar dentro do padrão estabelecido pelo Ministério da Saúde e a Organização Mundial da Saúde, observouse que o atendimento pré-natal não está seguindo os protocolos ou manuais que regulamentam os procedimentos e exames realizados. ${ }^{2-4}$ Exames como os de sangue, urina, ultra-sonografia, altura uterina, medida da pressão arterial, peso da gestante e ausculta do bebê foram referidos como tendo sido realizados em quase todas as gestantes, nesse estudo. Contudo, procedimento importante como exame das mamas foi efetuado em menos da metade da amostra.

Santos et al.,14 Victora et al.,15 e Costa et al.,16 na comparação de duas coortes com respectivas tendências e diferenciais, reforçam a mesma teoria e salientam que a menor freqüência relatada pelas mães quanto ao exame das mamas pode estar assinalando a menor probabilidade de orientação para a amamentação.

Orientações sobre o parto foram repassadas a $61 \%$ das gestantes, mas, orientações sobre amamen- tação a apenas $48 \%$. Kramer et al. 17 afirmam que a promoção ao aleitamento materno reduz o risco de exposição a doenças gastrointestinais, problemas respiratórios e eczemas ectópicos, entre outras ocorrências, no primeiro ano de vida. Estudos demonstram que toda promoção, aconselhamento, assim como campanhas do governo, como Hospital Amigo da Criança, contribuem significativamente para o aumento dos índices de amamentação, reduzindo substancialmente os riscos de doenças e morte no primeiro ano de vida. Ressalta-se, assim, a importância da educação em saúde, que deve ser repassada com prioridade a todas as gestantes. ${ }^{1-4}$

Esta pesquisa encontrou alta cobertura vacinal (imunização antitetânica), o mesmo ocorrendo com a suplementação de ferro, visto que $78 \%$ das gestantes usaram medicação contra anemia. Observouse, ainda, que metade das gestantes referiu ter corrimento durante a gestação, mas apenas 33\% da amostra foram examinadas por esse motivo, indicando falhas no manejo da situação, apesar de saberse que problemas ginecológicos não tratados adequadamente podem levar ao parto prematuro e a complicações na gravidez. $18-20$

Ressalta-se o fato de $62 \%$ das gestantes não terem feito teste de tolerância à glicose. Segundo o Ministério da Saúde, 1 o diabetes gestacional (DG) é um dos fatores de risco para o parto prematuro e de complicações durante o pré-natal, como parto cesário, problemas respiratórios para o bebê, entre outras, elevando os índices de morbimortalidade perinatal. O rastreamento nas primeiras consultas de PN é fator decisivo para o cuidado especial e para intervenções que deverão ser dispensadas a essa gestante de alto risco.1-3,10

Evidenciou-se um risco seis vezes maior de um pré-natal inadequado nas famílias com renda mensal até um salário mínimo. $\mathrm{O}$ mesmo achado também foi referido por Tomasi et al., ${ }^{5}$ Barros et al., ${ }^{6}$ e Barros et al., ${ }^{8}$ nas coortes de acompanhamento de 1982, 1993 e 2004 .

A pouca escolaridade materna também é um fator de risco para a realização de um pré-natal inadequado. Halpern et al. 4 indicam que mulheres decididas a fazer consultas pré-natais, com regularidade, possivelmente pertencem a um grupo com maior consciência da importância de medidas preventivas em saúde, como não fumar, não ingerir álcool, alimentar-se adequadamente e evitar infecções. Não ter trabalho remunerado, assim como ser fumante, não possuir companheiro e ser grande multípara aumentou o risco para a realização de um pré-natal inadequado. 
Os resultados do presente estudo confirmam a "lei da assistência inversa", segundo a qual a disponibilidade de bons serviços médicos tende a ser inversamente proporcional às necessidades da população atendida. Os grupos mais vulneráveis da sociedade, de menor renda e escolaridade são justamente os que têm menor acesso aos serviços de saúde e, possivelmente, os piores desfechos gestacionais. ${ }^{15,19-23}$

Esse estudo evidencia a perpetuação de achados de outras pesquisas realizadas em Pelotas, onde foi constatado que, apesar da ampla cobertura pré-natal, com postos de saúde e hospitais, o cuidado médico oferecido às gestantes tem pouca qualidade, pois existem falhas no atendimento e os protocolos não estão sendo seguidos. Esforços devem ser feitos para melhorar a qualidade da atenção às futuras mães,

\section{Referências}

1. Brasil. Ministério da Saúde. Programa de Humanização no Pré-natal e Nascimento. Brasília, DF; 2000. [monografia online]. [acesso em: 8 jun. 2004]. Disponível: http:// dtr2001.saude.gov.br/sps/areastecnicas/mulher/cartilha.htm

2. FEBRASGO (Federação Brasileira das Sociedades de Ginecologia e Obstetrícia). Projeto Diretrizes 2001. [monografia online]. [acesso em 17 maio 2001]. Disponível: http://www. projetodiretrizes.org.br

3. Brasil. Ministério da Saúde. Secretaria de Políticas de Saúde. Departamento de Gestão de Políticas Estratégicas. Gestação de alto risco: manual técnico. 3. ed. Brasília, DF, 2000.

4. Halpern R, Barros F, Victora C, Tomasi E. Atenção prénatal em Pelotas, Rio Grande do Sul, Brasil, 1993. Cad Saúde Pública. 1998; 14: 487-92.

5. Tomasi E, Barros, Victora C. As mães e suas gestações: comparação de duas coortes de base populacional no sul do Brasil. Cad Saúde Pública. 1996; 12: 21-5.

6. Barros F, Victora C, Tomasi E. Saúde materno-infantil em Pelotas, Rio Grande do Sul, Brasil: principais conclusões da comparação dos estudos das coortes de 1982 e 1993. Cad Saúde Pública. 1996; 12: 87-92.

7. Silveira D, Santos I, Costa JD. Atenção pré-natal na rede básica: uma avaliação da estrutura e do processo. Cad Saúde Pública. 2001; 17: 131-9.

8. Barros FC, Victora C, Barros A. The challenge of reducing neonatal mortality in middle-income countries: findings from three Brazilian birth cohorts in 1982, 1993, and 2004. Lancet. 2005; 365: 847-54.

9. Menezes AMB, Barros F, Victora C. Fatores de risco para mortalidade perinatal em Pelotas, RS, 1993. Rev Saúde Pública. 1998; 32: 209-16. motivando e capacitando os profissionais para a prática da saúde, com equipes de trabalho multidisciplinares, garantindo e fiscalizando a realização dos protocolos estabelecidos pelo Ministério da Saúde.13 Ressalta, também, o fato de que as adolescentes têm um risco maior de realizarem um pré-natal inadequado; sugere-se novos estudos explorando esses dados, que são indispensáveis para traçar um perfil dessa parcela importante e representativa da sociedade.

Novos estudos investigando a qualidade da atenção pré-natal de Pelotas, assim como a educação médica continuada, com o cumprimento das normas técnicas pré-estabelecidas e o melhor gerenciamento dos recursos de saúde disponíveis são as ferramentas para reverter esse quadro e oferecer uma assistência eqüitativa e de qualidade a todas as gestantes.

10. Coimbra LC, Silva AM, Mochel E. Fatores associados à inadequação do uso da assistência pré-natal. Rev Saúde Pública. 2003; 37: 456-62.

11. Hobel CJ, Hyverinem MA, Okada DM, Oh W. Prenatal and intrapartum high-risk screening, prediction of the high-risk neonate. Am J Obstetr Gynecol. 1973; 117: 1-9.

12. Zambonato AMK, Pinheiro RT, Horta BL, Tomasi E. Fatores de risco para nascimento de crianças pequenas para idade gestacional. Rev Saúde Pública. 2004; 38: 24-9.

13. CEBRID (Centro Brasileiro de Informações sobre Drogas Psicotrópicas). Utilização e consumo de tabaco. [monogafia online]. [acesso em: 9 fev. 2005]. Disponível: http:// www.unifesp.br/dpsicobio/cebrid/folhetos/tabaco (2005 fev 9).

14. Santos IS, Baroni RC, Minotto I, Klumb AG. Critérios de escolha de postos de saúde para acompanhamento pré-natal em Pelotas, RS. Rev Saúde Pública. 2000; 34: 603-9.

15. Victora CG, Barros F, Vaughan JP. Epidemiologia da desigualdade: um estudo longitudinal de 6.000 crianças brasileiras. São Paulo: Hucitec; 1989.

16. Costa JD, Victora CG, Barros F. Assistência médica materno-infantil em duas coortes de base populacional no Sul do Brasil: tendências e diferenciais. Cad Saúde Pública. 1996; 12: 59-66.

17. Kramer MS, Chalmers B, Hodnett ED. Promotion of breastfeeding intervention trial (PROBOT. JAMA. 2001; 285: 2431.

18. Silveira D, Santos I. Adequação do pré-natal e peso ao nascer: uma revisão sistemática. Cad Saúde Pública. 2004; 20: $1160-8$.

19. Leal MC, Gama SGN, Cunha CB. Uso do índice de Kotelchuck na avaliação da assistência pré-natal e sua relação com as características maternas e o peso do recémnascido no Município do Rio de Janeiro. Cad Saúde Pública. 2004; 20 (Sup1): 563-572. 
20. Silva AA, Coimbra LC, Raimundo AS. Perinatal health and mother-child health care in the municipality of São Luis, Maranhão State, Brazil. Cad Saúde Pública. 2001; 17 : 1413-23.

21. Rodrigues Filho J, Costa W, Leno GM. Determinantes de utilização do cuidado pré-natal entre famílias de baixa renda no Estado da Paraíba, Brasil. Rev Saúde Pública. 1994; 28: 204-9.

Recebido em 8 de novembro de 2007

Versão final apresentada em 2 de agosto de 2008

Aprovado em 9 de setembro de 2008
22. Moura ERF, Holanda FJR, Rodrigues MSP. Avaliação da assistência pré-natal oferecida em uma microrregião de saúde do Ceará, Brasil. Cad Saúde Pública. 2003; 19: 17919.

23. Kotelchuck M, Schwartz JB, Anderka MT. WIC Participation and pregnancy outcomes: Massachusetts Statewide Evaluation Project. Am J Public Health. 1984; 74: 1086-1092. 